\title{
La faune de la grotte Chauvet (Vallon-Pont-d'Arc, Ardèche) : présentation préliminaire paléontologique et taphonomique
}

The large fauna of Chauvet cave (Vallon-Pont-d'Arc, Ardèche): preliminary paleontological and taphonomic presentation

\section{Michel Philippe et Philippe Fosse}

\section{OpenEdition \\ Journals}

Édition électronique

URL : http://journals.openedition.org/paleo/1265

DOI : $10.4000 /$ paleo. 1265

ISSN : 2101-0420

Éditeur

SAMRA

Édition imprimée

Date de publication : 1 décembre 2003

Pagination : $123-140$

ISSN : 1145-3370

Référence électronique

Michel Philippe et Philippe Fosse, « La faune de la grotte Chauvet (Vallon-Pont-d'Arc, Ardèche) :

présentation préliminaire paléontologique et taphonomique », PALEO [En ligne], 15 | 2003, mis en ligne le 05 août 2010, consulté le 07 juillet 2020. URL : http://journals.openedition.org/paleo/1265 ; DOI : https://doi.org/10.4000/paleo.1265

Ce document a été généré automatiquement le 7 juillet 2020

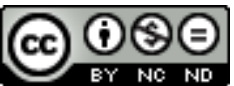

PALEO est mis à disposition selon les termes de la licence Creative Commons Attribution - Pas d'Utilisation Commerciale - Pas de Modification 4.0 International. 


\section{La faune de la grotte Chauvet (Vallon-Pont-d'Arc, Ardèche) : présentation préliminaire paléontologique et taphonomique}

The large fauna of Chauvet cave (Vallon-Pont-d'Arc, Ardèche): preliminary paleontological and taphonomic presentation

Michel Philippe et Philippe Fosse

En hommage à Philippe Morel

\section{Introduction}

1 La grotte Chauvet est célèbre pour l'excellente exécution (mais aussi conservation) de ses œuvres pariétales (Clottes 2001). Ce gisement est également important pour son exceptionnelle richesse, aussi bien qualitative que quantitative, des témoins paléobiologiques (vestiges osseux et témoins d'activité) d'origine humaine et surtout animale, au sol (empreintes, bauges) et sur paroi (tracés digités / polis, griffades). Un premier bilan paléozoologique et anthropozoologique est ici présenté concernant les vertébrés identifiés pour l'ensemble des zones accessibles de la cavité.

2 Les techniques d'étude préconisées pour une conservation optimale de l'ensemble du registre archéologique reposent sur la non manipulation des pièces paléontologiques et une circulation volontairement limitée dans la cavité. Par conséquent, de nombreuses déterminations spécifiques et/ou anatomiques ont été effectuées à distance (déterminations au moyen de jumelles ou de photos prises au téléobjectif pour les pièces inaccessibles ; premiers essais de scannage tridimensionnel des amas ${ }^{1}$. Les prises de mesure ne sont, méthodologiquement parlant, guère comparables avec ce qui peut être fait classiquement sur des échantillons en laboratoire. L'inaccessibilité des pièces 
et leur manipulation extrêmement restreinte n'autorisent que peu d'informations ostéométriques, néanmoins nécessaires à toute étude paléontologique. Les plans de mesurage "standard" (Desse et al. 1986 pour l'ensemble des espèces, Torres PerezHidalgo 1988; Tsoukala et Grandal d'Anglade 2002 pour les ursidés), ont été utilisés mais ont souvent dû être adaptés ou modifiés. A terme, cela nécessitera d'établir un corpus de comparaison avec des échantillons disponibles dans des musées.

La grotte, d'un développement de 500 mètres environ (fig.1), a été délimitée en secteurs paléontologiques (environ 150 loci). Ces secteurs renferment quelques ou, le plus souvent, plusieurs dizaines d'ossements (amas). Cette répartition résulte de l'action combinée et diachronique des ours (modifications de leurs habitats, cf. Koby 1953), de l'homme (manipulations d'ossements) et de l'eau (charriage). Il y a une nette dichotomie entre les premières salles (Salle Brunel, Salle des Bauges) et les salles du fond (Salle Hillaire et au delà); cette différence, qui est en partie due à une configuration topographique différentielle (grandes salles / galeries; sol argileux / calcitation importante), porte non seulement sur la concentration du matériel paléontologique (faible / forte) mais aussi sur son degré de préservation (pas très bon (lessivage, recristallisation) / (très) bon (calcité)). Ceci pose des problèmes d'interprétation globale de la cavité relative à sa fonction (zonations comportementales ?) et à ses modalités d'occupation (syn/diachronique; ours / homme?). Sur les sols argileux (Salle des Bauges, Galerie des Mégacéros...), la préservation des ossements est globalement moins bonne que dans les secteurs calcités (importantes destructions physico-chimiques, produites par les conditions de milieu (forte humidité, teneur élevé en $\mathrm{CO} 2$ de l'air). Dans le premier cas, les pièces sont facilement identifiables mais inaccessibles et fragmentées, dans le second au contraire, facilement accessibles mais encroûtées jusqu'à disparaître dans les planchers stalagmitiques et non déterminables spécifiquement ou anatomiquement ou non mesurables. 
Figure 1 - Grotte Chauvet : sectorisation de la grotte. Relevés topographiques : Le Guillou et Maksud (2001).

Figure 1 - Chauvet cave: usual names of different galleries.

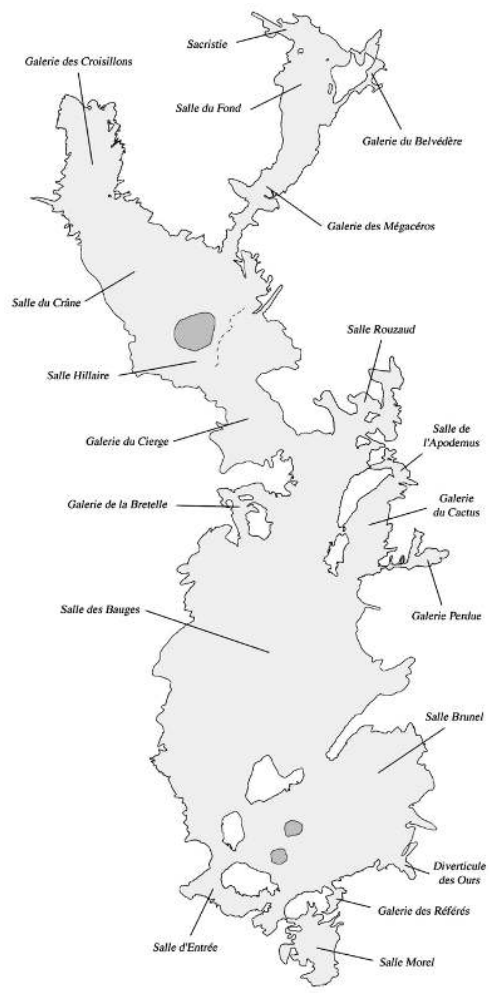

4 Les contraintes de préservation des sols et des parois affectent donc les méthodes classiques d'étude (biostratigraphie, ostéométrie (multivariée), morphologie comparée des séries jugales, des surfaces articulaires ...) mais permettent en revanche d'esquisser, par des observations de contrôle pouvant être réitérées, des relations paléobiologiques et des processus taphonomiques par contrôles permanents des ensembles osseux à partir de l'ensemble des témoins archéologiques (relations prédateurs-proies d'après les empreintes, cf. Garcia 2001 ; rôle joué par les agents biologiques et abiotiques dans la formation des amas d'ossements...).

\section{1 - Présentation générale de la faune}

5 La faune identifiée (Philippe et Fosse $2001 ;$ tabl. 1) peut être rapportée à au moins neuf espèces de carnivores (Ursus spelaeus, Ursus arctos, Panthera pardus, Felis silvestris, Canis lupus, Vulpes vulpes, Martes martes, Martes foina, Crocuta crocuta spelaea), cinq d'ongulés (Bos ou Bison, Capra ibex, Capreolus capreolus, Cervus elaphus, Equus caballus), trois de rongeurs (Apodemus silvaticus, Eliomys quercinus, Microtus nivalis), un chiroptère (Myotis myotis et quelques os d'un Chiroptère indéterminé), cinq espèces d'oiseaux (Aquila chrysaetos, Pyrrhocorax graculus, Cinclus cinclus, turdidae sp. et un passériforme indéterminé) et un reptile (couleuvre ?). Cette richesse n'est apparente que du point de vue taxonomique et semble correspondre à des fréquentations diachroniques (Pléistocène supérieur et Holocène) et différenciées (volontaires (carnivores) ou involontaires (ongulés = proies ?, oiseaux ?) de la cavité par les différents taxons. La diversité faunique (en nombre de taxons) est plus forte dans les zones proches de l'entrée (notamment dans l'Eboulis, au sommet de la Salle des Bauges) que dans les 
zones profondes. Dans ces derniers secteurs, la distribution est presque monospécifique (Ours des cavernes). Du point de vue paléoécologique, l'Ours constitue 99,9\% de la faune, conformément à ce qui est attendu d'une grotte à Ours des cavernes (Fosse et al. 2002; Philippe 1993). Aucune espèce “ froide " n'est présente dans le registre paléontologique contrairement à certaines représentations pariétales (Rangifer tarandus ( $\mathrm{n}=12$ cf. Baffier et Feruglio 2001a), Ovibos moschatus ( $\mathrm{n}=2$, cf. Le Guillou 2001), Coelodonta antiquitatis ( $\mathrm{n}=65$, cf. Aujoulat et Guerin 2001), Mammuthus primigenius ( $\mathrm{n}=66$, cf. Gely 2001). D'autres espèces (Panthera spelaea, Megaloceros giganteus...), relativement abondantes dans le bestiaire pariétal, font, à ce jour, défaut dans les ensembles paléontologiques.

\section{1 - La faune holocène}

6 La petite faune, présentant un degré de fossilisation moindre de celui des taxons assurément pléistocènes (Ursus), a été considérée comme holocène. Elle n'est pas très abondante mais est bien individualisée géographiquement et comprend (fig. 2) : des petits carnivores (martre et/ou fouine), quelques espèces de rongeurs (mulot, campagnol, lérot et loir), chauves-souris (le grand murin et restes isolés de chiroptères encore indéterminés), petits passereaux (le cincle plongeur, un petit Turdidae et un autre passereau indéterminé) et un serpent (couleuvre ?).

Figure 2 - Grotte Chauvet : répartition spatiale des espèces holocènes. Figure 2 - Chauvet cave : spatial distribution of holocene fauna.

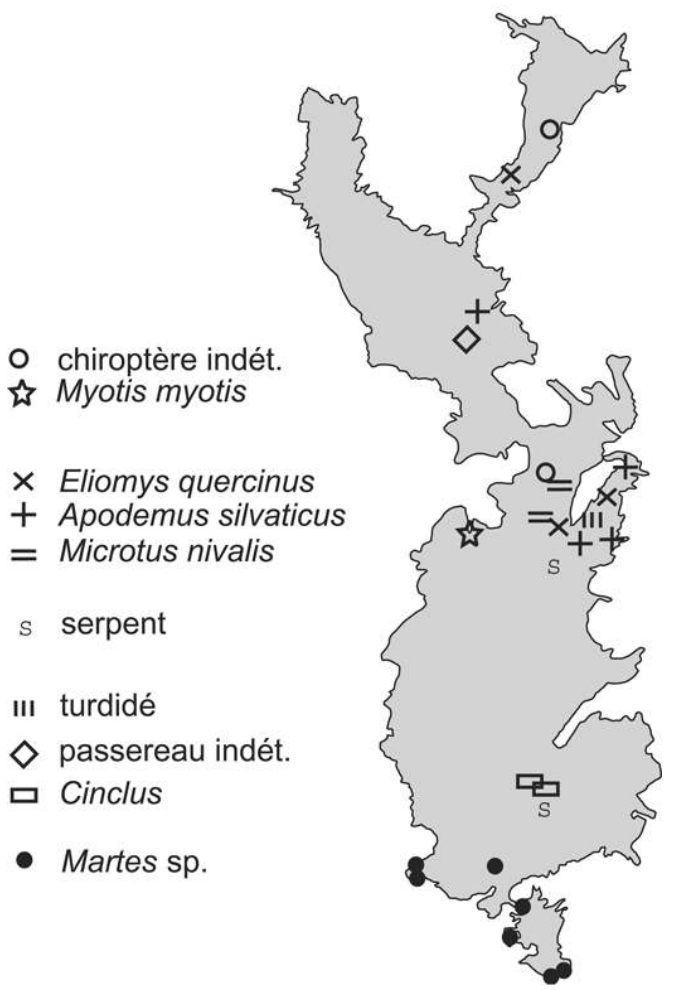

7 Huit squelettes plus ou moins complets de mustélidés, trouvés dans les zones proches de l'entrée et appartenant à de jeunes adultes morts durant leur hivernation, ont été dénombrés (trois individus dans ou à proximité de l'Eboulis d'entrée ; quatre individus dans la Salle Morel ; un individu dans la Galerie de la Lucarne). Par comparaison avec 
des données ostéomorphologiques des têtes osseuses (Rode et Didier 1944; Altuna 1973a), il est possible d'identifier, à partir des pièces accessibles et/ou mesurables, au moins une fouine et deux martres.

8 Les rongeurs sont peu abondants. Le mulot (Apodemus silvaticus) a été identifié dans la Galerie du Cactus (deux individus) et dans son prolongement (salle de l'Apodemus, un individu en cours de décomposition) ainsi que dans la salle Hillaire (bord du grand effondrement). Deux éléments de squelette de campagnol des neiges (Microtis nivalis ${ }^{2}$ ), espèce localement disparue ${ }^{3}$, ont été identifiés dans la Salle des Panneaux Rouges. Le lérot (Eliomys quercinus) a été trouvé dans la Galerie du Cactus (un individu), dans la Salle des Panneaux Rouges (un individu) et dans la Galerie des Mégacéros (un individu).

La fréquentation de la cavité par les chauves-souris est attestée sur les parois par de petites cupules de corrosion imprégnées d'argile ainsi que par une multitude de griffades (Salle Hillaire, Galerie des Mégacéros). De plus, quelques os isolés ont été repérés: quatre os d'aile dans la Galerie du Cactus, un radius dans la Salle de l'Apodemus, un humérus de grand murin, Myotis myotis ${ }^{4}$ sous le panneau de la Panthère, cinq os longs et une hémimandibule dans la Bretelle, un humérus peu avant le Seuil, trois os longs avant le grand " escalier" dans cette même galerie.

Les restes d'oiseaux ne sont pas abondants ${ }^{5}$. Indépendamment des deux espèces pléistocènes (cf. infra), quelques os de cincle plongeur (Cinclus cinclus, NMI $=2$ ) ont été trouvés dans la Salle Brunel, juste en aval du panneau du Sacré-Cœur. Quelques ossements d'un petit turdidé ont également été repérés dans la Galerie du Cactus. Enfin, un squelette complet et en parfaite connexion anatomique d'un petit passereau indéterminable car recouvert d'une pellicule calcitique est visible tout près du grand effondrement de la Salle Hillaire.

11 Au moins deux serpents ont été trouvés dans la grotte. De longs tronçons de squelette, avec plus d'une dizaine de vertèbres accompagnées éventuellement des côtes, ont été découverts dans la Salle Brunel (plusieurs tronçons vraisemblablement d'un même animal (couleuvre d'Esculape ${ }^{6}$, en aval du panneau du Sacré Cœur, échelonnés sur plus d'un mètre de longueur, dans un petit ravinement) et dans une niche rocheuse de la Galerie du Cactus, au-dessous du petit ours rouge de gauche.

12 La présence de cette faune peut être soit volontaire (martre, fouine, serpent) soit involontaire (oiseaux : accidents ?).

\section{2 - La faune pléistocène}

13 Les vestiges osseux pouvant être rattachés aux espèces ayant fréquenté la cavité au cours du Pléistocène sont très abondants et appartiennent majoritairement à l'Ours des cavernes. Bien que marginales en nombre de restes, les autres espèces de vertébrés posent d'importantes questions d'ordre paléobiologique et taphonomique (relations des différentes espèces: proies/prédateurs, modalités d'apport dans la cavité) en relation avec les premiers résultats des autres disciplines (notamment l'ichnologie).

\section{Le Cheval (Equus caballus)}

14 Le Cheval n'est identifié que par deux restes (cf. localisation in figure 3) : une molaire supérieure, au bas de la Salle des Bauges, à plusieurs mètres du cheminement autorisé et une extrémité distale de métapode découverte dans un des nombreux amas de 
l'Eboulis d'entrée. La présence de ces vestiges est sans doute accidentelle. Aucune trace d'origine anthropique ou carnivore n'a été décelée sur la seule pièce accessible.

\section{Le Chevreuil (Capreolus capreolus)}

Les restes de Chevreuil sont un peu moins rares et sont localisés en deux points de la grotte (fig. 3) : sur le sommet et au bas de l'Eboulis qui a colmaté l'entrée paléolithique ainsi que dans les gours de la "loggia ", à l'entrée de la Salle Morel. Chaque salle semble renfermer les restes d'un individu distinct.

Figure 3 - Grotte Chauvet : répartition spatiale des espèces pléistocènes marginales. Figure 3 - Chauvet cave : spatial distribution of marginal pleistocene fauna.

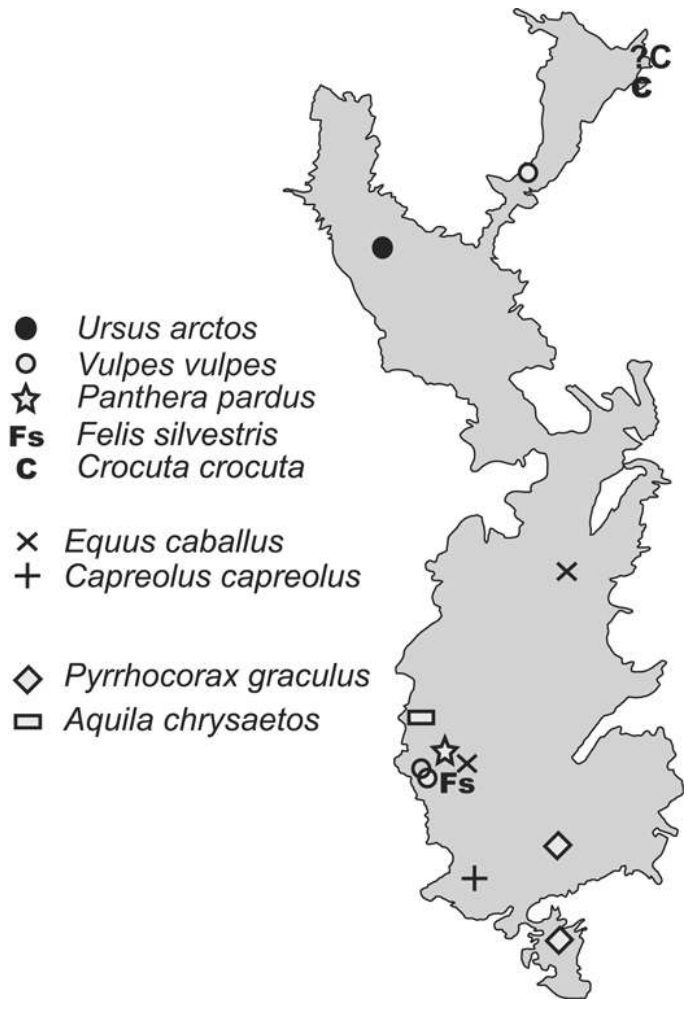

$\mathrm{Au}$ sommet de l'Eboulis d'entrée et strictement en surface, une couche de calcite recouvrant une scapula a été datée de $12985 \pm 85$ BP (LY-10489). Au bas de cet éboulis, plaqués contre la paroi rocheuse qui sépare la Salle Brunel de la Salle des Bauges, se trouvent un métatarse et un radius-ulna qui pourraient être appariés à la scapula.

Dans les gours de la " loggia " qui domine la Salle Morel, tout près de l'étroite chatière d'accès, plusieurs éléments d'un même membre pelvien appartenant à un second chevreuil ont été identifiés : un tibia, un métatarse, l'ensemble des os du tarse, deux premières phalanges, une deuxième et une troisième phalanges. Aucune trace d'origine biologique n'a pu être relevée sur ces pièces.

\section{Le Bouquetin (Capra ibex)}

18 Sa présence est attestée par des vestiges osseux dans les salles proches de l'entrée paléolithique (Salle Morel, Eboulis d'entrée, Salle Brunel ; fig. 4) et plus curieusement 
par de nombreuses empreintes dans les parties profondes de la cavité (Galerie des Mégacéros, Sacristie : cf. Garcia 2001).

Figure 4 - Grotte Chauvet : répartition spatiale des ossements de bouquetin.

Figure 4 - Chauvet cave : spatial distribution of ibex bones.

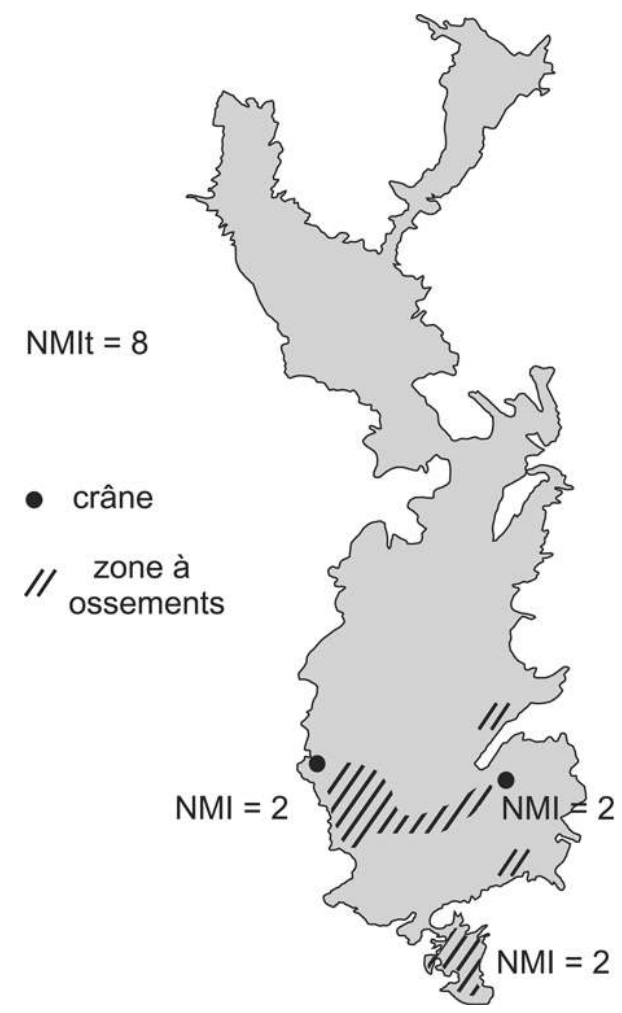

Dans la Salle Morel, des restes d'au moins deux individus ont été recensés (un subadulte et un adulte), représentés par des mandibules, des os longs et des éléments de la colonne vertébrale. Dans l'Eboulis d'entrée, l'arrière-crâne d'un jeune animal, plusieurs os longs, des hémi-mandibules à branches horizontales fracturées longitudinalement (origine anthropique?) et des portions de métapodes ont été identifiés soit au sein d'amas d'ossements d'ours, soit isolés. Dans la salle Brunel, un crâne appartenant à un sujet mâle adulte a été charrié par ruissellement. Il repose sur sa face frontalooccipitale et sur les chevilles osseuses, bien conservées mais scellées au sol concrétionné par une mince pellicule de calcite.

\section{La Panthère (Panthera pardus)}

Une extrémité distale d'humérus a été découverte dans l'Eboulis d'entrée (fig. 5). Sa présence, bien que toujours rare, est attestée localement dans d'autres sites, comme l'aven du Marteau, profond aven situé sur la même commune de Vallon-Pont d'Arc, au fond duquel a été recueillie une canine de ce félin parfaitement conservée ${ }^{7}$. 
Figure 5 - Ostéométrie comparée (DTd) des humérus de panthères et lions pléistocènes avec l'échantillon de Chauvet.

Figure 5 - Comparative measurements of humerus (dTD) between pleistocene leopard and lion with Chauvet sample.
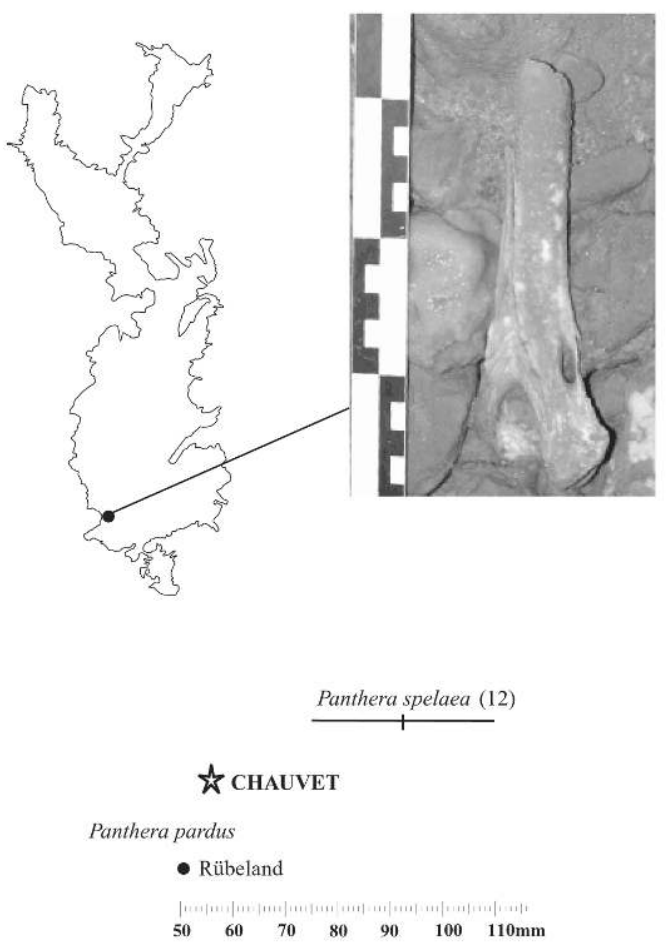

\section{Le Chat sauvage (Felis silvestris)}

21 L'Eboulis d'entrée a livré une seconde extrémité distale d'humérus de félidé, de très petite taille et de même degré de conservation que les ossements des autres espèces pléistocènes. Son attribution au Chat sauvage a été retenue.

\section{L'Hyène tachetée fossile (Crocuta crocuta spelaea)}

Ce carnivore n'a été identifié que récemment par un troisième métacarpien, situé dans la partie la plus profonde et la moins accessible de la cavité (petite niche dans le Belvédère). Un peu plus loin, gît dans une épaisse couche d'argile meuble un crâne dont les seules caractéristiques morphologiques visibles (boîte crânienne globuleuse, sommet du chignon occipital saillant, crête sagittale apparemment bien développée) pourrait également suggérer cette espèce (fig. 6). Cette nouvelle découverte pose un intéressant problème taphonomique, concernant les associations fauniques (proies/ prédateurs ; relations inter-prédateurs). 
Figure 6 - Ostéométrie comparée (L totale x DT bizygomatique) des crânes d'hyènes et loups fossiles avec l'échantillon de Chauvet.

Figure 6 - Comparative measurements (total length $x$ bizygomatic Td) of fossil wolf and spotted hyena skulls with Chauvet sample.
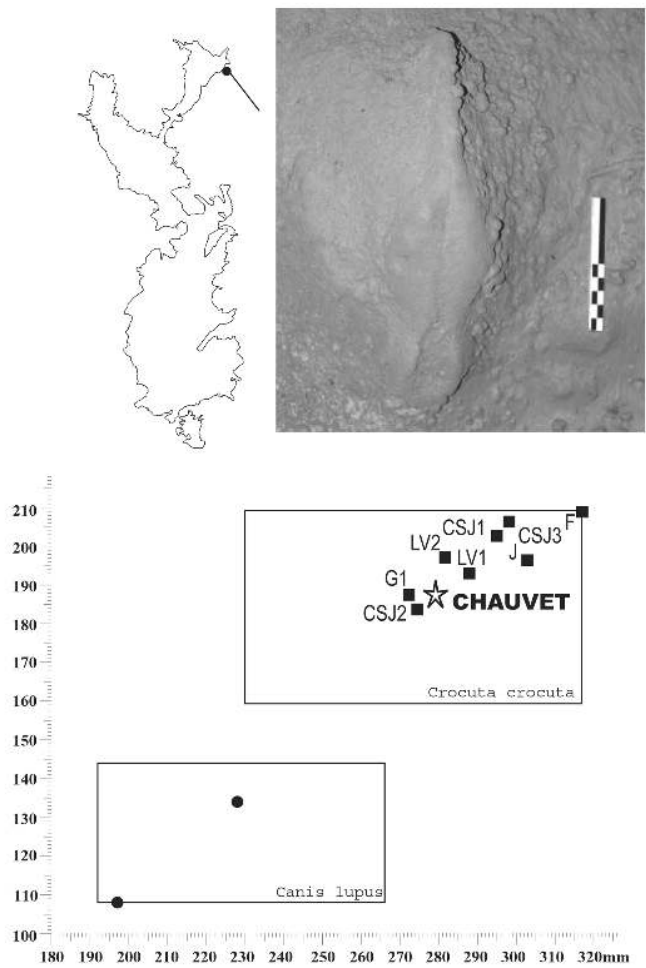

\section{Le Renard roux (Vulpes vulpes)}

Le Renard a été identifié dans l'Eboulis d'entrée (une carnassière supérieure) et dans la Galerie des Mégacéros. Dans ce secteur (fig. 3), un squelette pratiquement complet dont les éléments sont éparpillés sur environ un mètre carré a été identifié à proximité des foyers (palier qui précède la Salle du Fond). L'attribution à Vulpes vulpes tient à la robustesse des éléments squelettiques; la carnassière inférieure a un diamètre mésiodistal de 16,2 $\mathrm{mm}^{8}$, dimension conforme aux renards roux pléistocènes (fig. 7). L'état de dégradation avancé des ossements ainsi que leur enfouissement ne permet, à ce jour, d'établir de lien géo-chronologique entre cet animal et les structures de combustion proches. 
Figure 7 - Grotte Chauvet : diamètre mésio-distal (en $\mathrm{mm})$ de la carnassière inférieure $(\mathrm{M} / 1)$ de renard provenant de la galerie du Megacéros.

Figure 7 - Chauvet cave: mesio-distal diameter (in $\mathrm{mm}$ ) of the fox lower carnassial found in galerie du Mégacéros. Comparisons with modern and fossil V. vulpes (lines) and A. lagopus (dotted lines).

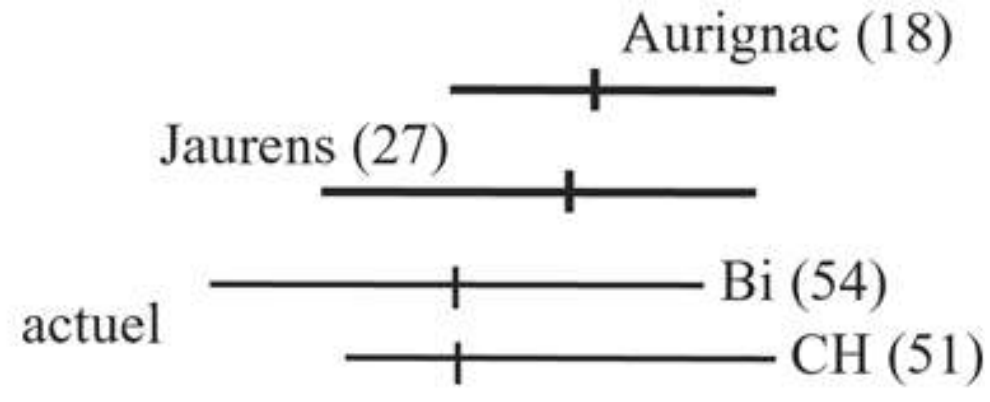

\section{Ł CHAUVET}

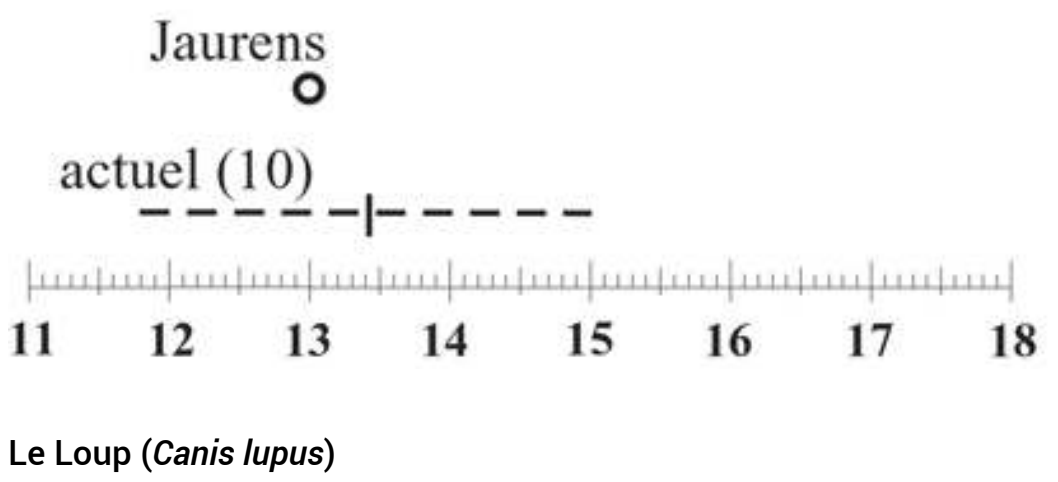

Les vestiges appartenant à cette espèce sont peu nombreux mais correspondent cependant à au moins six individus (fig. 8). Les loups ont fréquenté et exploré l'ensemble du développement karstique (traces de leur(s) passage(s) jusque dans les parties les plus profondes de la grotte; Garcia 2001). Des restes d'au moins deux individus (deux atlas, une hémi-mandibule, un radius, un ulna, un fémur) ont été trouvés dans l'Eboulis d'entrée et dans le sommet de la Salle des Bauges. D'autres pièces, isolées ont été identifiées dans différents secteurs (un métatarse dans la Salle Brunel), avec tout particulièrement deux crânes complets, l'un, de faibles dimensions (femelle ?, cf. infra) charrié à la faveur d'un ravinement dans la zone d'épandage du bas de la salle Brunel, l'autre, beaucoup plus robuste (mâle ?), dans la niche terminale du Belvédère. Dans la salle des Panneaux Rouges, au niveau du panneau des Mains Positives, trois os seulement ont été repérés mêlés à un amas d'ossements d'ours : une première phalange et deux vertèbres atlas. Le crâne de la Salle Brunel mesure $230 \mathrm{~mm}$ de longueur. Ces dimensions, modestes pour un spécimen pléistocène (fig. 9), suggère, en dépit d'échantillons populationnels stricto sensu numériquement faibles, une appartenance à un sujet femelle. 
Figure 8 - Grotte Chauvet : répartition spatiale des ossements de loup. Figure 8 - Chauvet cave: spatial distribution of wolf bones.

NMIt $=6$

- crâne

// zone à

ossements

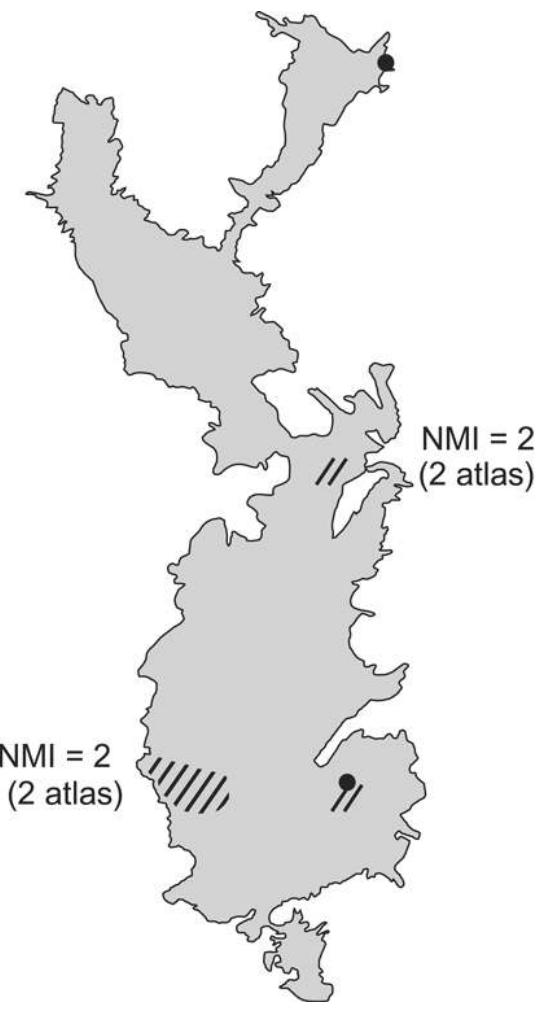

Figure 9 - Ostéométrie comparée ( $L$ totale) des crânes de loups et chiens, actuels et fossiles. Figure 9 - Comparative osteometry (total lenght) of modern and fossil wolf and dog skulls.
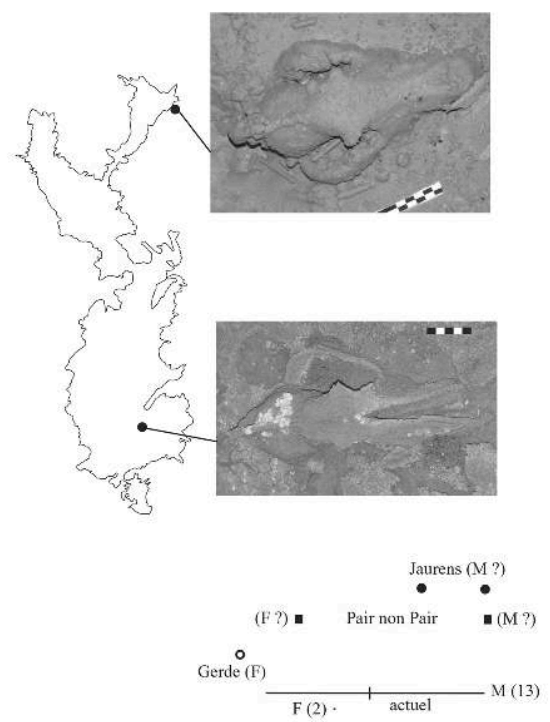

Ł CHAUVET

Canis familiaris (11)

Canis hupus

$\begin{array}{lllllllllllll}150 & 160 & 170 & 180 & 190 & 200 & 210 & 220 & 230 & 240 & 250 & 260 & 270 \mathrm{~mm}\end{array}$ 


\section{L'Ours brun (Ursus arctos)}

L'un des nombreux crânes d'ours repérés dans la Salle du Crâne diffère de tous les autres par son faible diamètre bizygomatique et le bombement très peu marqué de l'os frontal. Bien que la région frontale de la boîte crânienne soit un peu endommagée, le raccordement des crêtes frontales avec la crête sagittale se fait très loin en arrière. Avec une longueur totale de $375 \mathrm{~mm}$ pour un diamètre transverse bizygomatique de $215 \mathrm{~mm}$, ce crâne peut être attribué à Ursus arctos, vraisemblablement de sexe mâle (fig. 10).

Figure 10 - Ostéométrie comparée du crâne d'Ursus arctos de la grotte Chauvet avec différents échantillons d'ours brun et d'ours des cavernes d'Europe occidentale.

Figure 10 - Comparative osteometry of U. arctos skull from Chauvet cave with other european brown bear and cave bear samples.

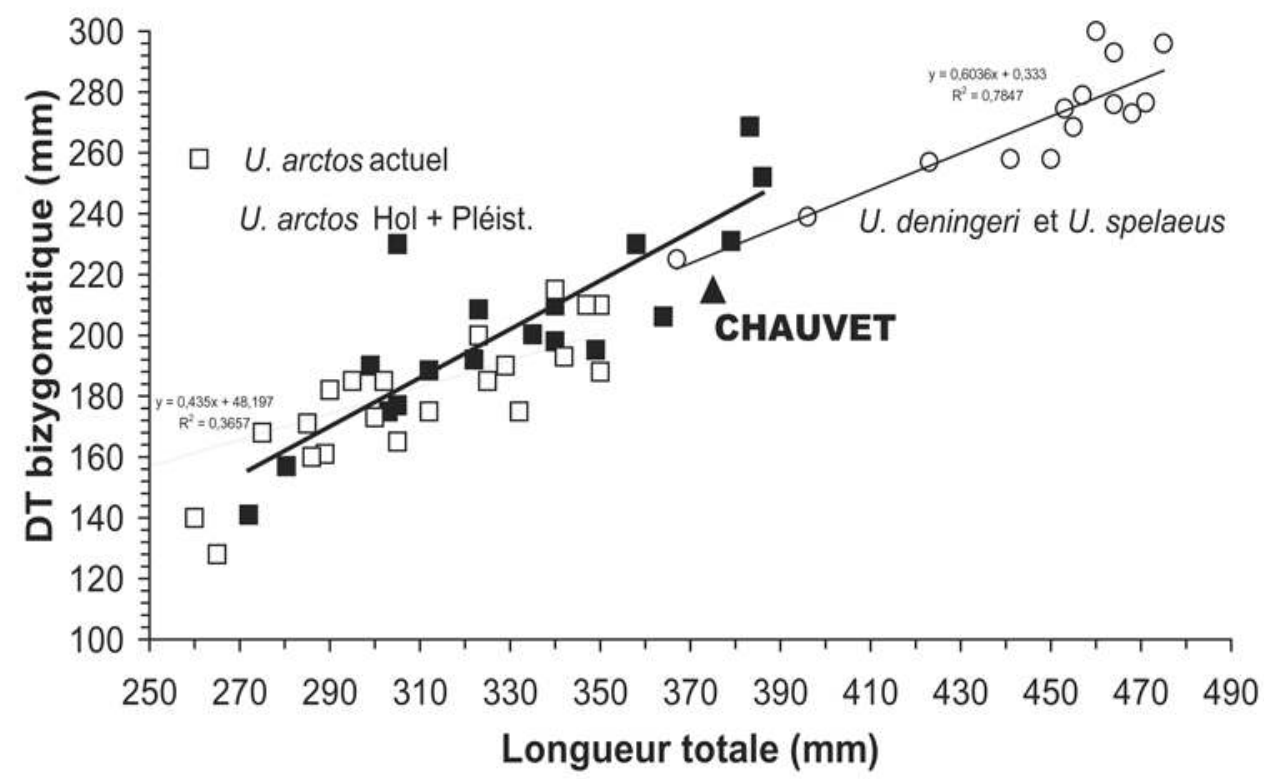

\section{L'Aigle royal (Aquila chrysaetos)}

Dans l'épandage de l'Eboulis du sommet de la Salle des Bauges (paroi de gauche), ont été repérés d'importants éléments d'un squelette de grand rapace ${ }^{9}$. Tous les éléments osseux sont englobés dans un concrétionnement. Si l'Aigle de Bonelli habite encore la région (c'est même l'un de ses derniers refuges), l'Aigle royal y a disparu certainement depuis l'Holocène. Parmi les ossements de ce squelette, ont été identifiés : le bec et des fragments du crâne, le bréchet, le sacrum, les deux hémi-coxaux, les deux fémurs, un humérus, un ulna et d'autres os recouverts par la calcite.

\section{Le Chocard à bec jaune (Pyrrhocorax graculus)}

Actuellement rassemblés dans les zones de moyenne montagne, les chocards sont généralement bien représentés dans les remplissages karstiques des périodes glaciaires, même à basse altitude (nichage dans des fissures des parois rocheuses). A la grotte Chauvet $^{10}$, seuls quelques restes ont été découverts, indépendamment de ceux, plus 
abondants, trouvés lors du déblaiement de l'entrée supérieure actuelle (sondage B. Gely 1998). Dans la Salle Brunel, un radius a été repéré dans un dépôt argileux entre les gros blocs rocheux qui occupent la partie droite de la salle (secteur 1-4D). Dans les gours de la "loggia " qui domine la Salle Morel, d'autres os (deux ulnas, deux métatarses et une troisième phalange) ont vraisemblablement été déposés par ruissellement. Il semble bien que la grotte n'ait pas été véritablement occupée par les chocards qui devaient nicher dans d'autres anfractuosités dans les falaises des gorges de l'Ardèche. La présence de ces quelques os est sans doute accidentelle.

\section{3 - L'Ours des cavernes (Ursus spelaeus)}

L'espèce animale la mieux représentée est incontestablement l'ours des cavernes. De très abondants vestiges osseux jonchent le sol et d'imposants témoins d'activité (empreintes, bauges, griffades, polis des pieds de paroi) sont visibles dans tous les secteurs de la cavité.

Plus de 130 amas d'ossements d'ours ont été inventoriés, depuis l'entrée (Salles Morel et Brunel, Eboulis d'entrée) jusqu'aux parties terminales (Galerie des Croisillons, Belvédère, Sacristie). Grands axes de circulation (Salles des Bauges, Hillaire et du Crâne) ou galeries secondaires (Diverticule des Ours, Galerie du Cactus, Salle Rouzaud) en renferment d'importantes quantités. L'inventaire porte à plus de 3500 le nombre d'ossements d'ours répartis sur l'intégralité des zones accessibles (tabl. 1); ce nombre pourrait être significativement augmenté par l'étude de certaines zones, particulièrement riches en vestiges osseux mais inaccessibles (la " plage" de la Salle des Bauges; Salle Rouzaud ?). À ce jour, le nombre minimum d'individus est de 190 (crânes ; tabl. 2 ; fig. 11), conférant à cette grotte une place tout aussi privilégiée pour la paléobiologie ursine que pour l'art pariétal. De nombreuses grottes d'Europe renferment de telles quantités d'ossements, mais toujours en stratigraphie (Fosse et al. 2002). A proximité de la grotte Chauvet et tout au long des gorges de l'Ardèche, une vingtaine de cavités renfermant des vestiges d'ours des cavernes a été recensée. Certaines (grottes de Louoï, de Cayre-Cret, toutes deux sur le territoire de Vallon-Pontd'Arc) ont été exploitées dès la fin du XIXème siècle par les phosphatiers. Parmi les grottes à ossements " humatiles ", peuvent êtres citées la grotte du Pas-de-Joulié (Gard), qui semble avoir contenu d'aussi importantes séries d'ours que la grotte Chauvet (son sol était, parait-il, recouvert de centaines de squelettes d'ours des cavernes, vandalisés en quelques jours) et la grotte de La Balme à Collomb (massif de Chartreuse, Savoie) qui recelait plusieurs milliers d'ossements éparpillés sur un sol d'environ 3000 mètres carrés (Philippe 1993).

Tableau 1 - Grotte Chauvet : nombre d'ossements, par espèce mammalienne, identifiés dans les différents secteurs accessibles.

Tableau 1 - Chauvet cave: number of bones, for each mammalian species, identified in different accessible places of the cave.

\begin{tabular}{|l|l|l|l|l|l|l|l|l|l|l|l|l|}
\hline Secteur & Ours & Loup & Renard & Hyène & $\begin{array}{l}\text { Pant- } \\
\text { hères }\end{array}$ & $\begin{array}{l}\text { Chat } \\
\text { sauv. }\end{array}$ & Martre & $\begin{array}{l}\text { Bou- } \\
\text { quetin }\end{array}$ & $\begin{array}{l}\text { Che- } \\
\text { vreuil }\end{array}$ & $\begin{array}{l}\text { Cervidé } \\
\text { Gd } \\
\text { bovid }\end{array}$ & Cheval \\
\hline $\begin{array}{l}\text { Salle } \mathrm{Ph} \\
\text { Morel }\end{array}$ & 20 & & & & & & 108 & 10 & 11 & & & \\
\hline
\end{tabular}




\begin{tabular}{|c|c|c|c|c|c|c|c|c|c|c|c|c|}
\hline $\begin{array}{l}\text { Salle des } \\
\text { Bauges, } \\
\text { Eboulis }\end{array}$ & $\mid 1044$ & $\mid 12$ & 1 & & 1 & 1 & 75 & 37 & 3 & 1 & 1 & 1 \\
\hline $\begin{array}{l}\text { Salle } \\
\text { Brunel }\end{array}$ & $\mid 69$ & 2 & 1 & & & & & 18 & & & $1 ?$ & \\
\hline $\begin{array}{l}\text { Salle des } \\
\text { Bauges }\end{array}$ & $\mid$\begin{tabular}{||l} 
\\
|
\end{tabular} & & & & & & & 2 & & & & 1 \\
\hline Cactus & 72 & & & & & & & & & & & \\
\hline La Bretelle & 48 & & & & & & & & & & & \\
\hline $\begin{array}{l}\text { Panneaux } \\
\text { Rouges }\end{array}$ & 321 & 3 & & & & & & & & & & \\
\hline $\begin{array}{l}\text { Galerie du } \\
\text { Cierge, } \\
\text { Seuil }\end{array}$ & 47 & & & & & & & & & & & \\
\hline $\begin{array}{l}\text { Galerie du } \\
\text { Cierge }\end{array}$ & $\mid$\begin{tabular}{||l}
103 \\
\end{tabular} & & & & & & & & & & & \\
\hline \begin{tabular}{|l} 
Salle \\
Hillaire, à \\
g. effond.
\end{tabular} & $\mid$\begin{tabular}{||l}
$\mid$ \\
484
\end{tabular} & & & & & & & & & & & \\
\hline $\begin{array}{l}\text { Salle } \\
\text { Hillaire, } \\
\text { après } \\
\text { effond. }\end{array}$ & $\mid 157$ & & & & & & & & & & & \\
\hline \begin{tabular}{|l} 
Galerie \\
des \\
Mégacéros
\end{tabular} & $\mid 232$ & & 30 & & & & & & & & & \\
\hline $\begin{array}{ll}\text { Salle } & d u \\
\text { Fond } & \end{array}$ & $\mid 221$ & & & & & & & & & & & \\
\hline $\begin{array}{l}\text { Salle du } \\
\text { Fond, } \\
\text { Belvédère }\end{array}$ & 20 & 1 & & $\begin{array}{l}1 \\
(+1 ?)\end{array}$ & & & 10 & & & & & \\
\hline $\begin{array}{l}\text { Salle du } \\
\text { Crâne }\end{array}$ & 432 & & & & & & & & & & & \\
\hline \begin{tabular}{|l} 
Galerie \\
des \\
Croisillons
\end{tabular} & $\mid 129$ & & & & & & & & & & & \\
\hline TOTAL & 3703 & 18 & 32 & 1 & 1 & 1 & 193 & 67 & 14 & $\mid 1$ & 2 & $\mid 2$ \\
\hline
\end{tabular}


Tableau 2 - Grotte Chauvet : nombre de crânes d'ours des cavernes identifiés dans les différents secteurs accessibles.

Tableau 2 - Chauvet cave: number of cave bear skulls identified in different accessible places of the cave.

\begin{tabular}{|c|c|}
\hline Secteur & Crânes état (complet / fragment) \\
\hline Salle Ph Morel & $-/ 1$ \\
\hline Salle des Bauges, Eboulis & $10 / 5$ \\
\hline Salle Brunel & $2 / 4$ \\
\hline Salle des Bauges & $4 / 3$ \\
\hline Cactus & $2 / 2$ \\
\hline La Bretelle & \\
\hline Panneaux Rouges & $9 / 3$ \\
\hline Salle Rouzaud & $3 /-$ \\
\hline Galerie du Cierge, Seuil & $3 / 1$ \\
\hline Galerie du Cierge & $17 / 1$ \\
\hline Salle Hillaire, à g. effond. & $35 /-$ \\
\hline Salle Hillaire, in effond. & $9 /-$ \\
\hline Galerie des Mégacéros & $2 /-$ \\
\hline Salle du Fond & $13 /-$ \\
\hline Salle du Fond, Belvédère & $1 /-$ \\
\hline Salle du Crâne & $53 /-$ \\
\hline Galerie des Croisillons & $7 /-$ \\
\hline TOTAL & 190 \\
\hline
\end{tabular}


Figure 11 - Grotte Chauvet : dénombrement et répartition spatiale des crânes d'ours des cavernes. Figure 11 - Chauvet cave: counting and spatial distribution of cave bear skulls.

crâne visible mais inaccessible

15 nombre de crânes
visibles sur le sol
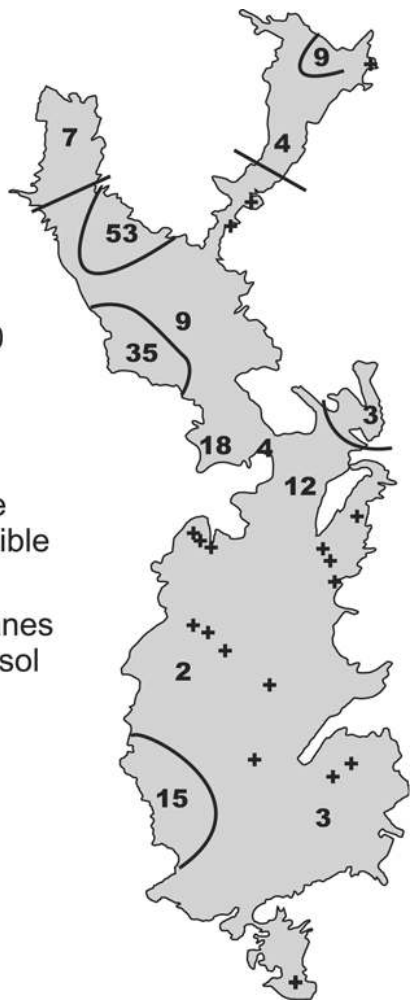

\section{2- Éléments de paléobiologie ursine}

Par son exceptionnelle palette de témoins relatifs au(x) séjour(s) des ours(es) dans la cavité, la grotte Chauvet offre l'opportunité d'appréhender l'éco-éthologie des ours des cavernes. Toutefois, les reconstitutions comportementales, basées sur des ostéocénoses "humatiles" (observations visuelles du matériel paléontologique jonchant le sol ou partiellement enfoui), n'est pas sans poser des problèmes qui pourraient être résolus par une opération de fouille stricto sensu (étude d'une taphocénose : durée d'occupation, synchronismes inter-assemblages, modalités démographiques ursines, remaniements post-dépositionnels...).

\section{1 - Chronologie des occupations}

S'il est évident que l'Ours des cavernes fut le premier et le plus important (en terme de durée de fréquentation) occupant de la cavité (peintures ou gravures recouvrant, dans leur majorité, des griffades ; abondance et diversité des témoins des activités ursines), il n'en demeure pas moins que l'absence d'une chronologie “ fine " limite, à ce jour, les observations d'ordre paléobiologique (fig. 12) ; les relations parois (griffades) - sols (ossements notamment) des activités ursines et anthropiques sont difficiles à saisir. De plus, les trois dates disponibles (Salle Philippe Morel, Salle Hillaire et Galerie des Mégacéros) font curieusement apparaître des occupations géochronologiques différenciées (fig. 10) et posent de nombreuses questions concernant : la durée globale estimée de la fréquentation de la cavité par les ours, la durée de mise en place des assemblages osseux les plus riches (Salle Hillaire, “ plage d'os " de la Salle des Bauges...), 
les modalités d'occupation (occupations synchroniques (nombre de phases d'occupation? phase d'occupation continue ?) ou diachroniques (sectorisation ? ${ }^{11}$ ), le fond de la grotte contient-il les éléments fauniques les plus anciens (sésamoïde d'ours daté de 31020 dans la Galerie du Mégacéros), phase terminale de fréquentation de la cavité par l'ours? (problèmes liés à l'extinction de cette espèce qui se raréfie entre 20 et 18000 BP en Europe ; cf. Fosse et al. 2001) ?

Figure 12 - Grotte Chauvet : datations ${ }^{14} \mathrm{C}$ disponibles pour Ursus spelaeus. Figure 12 - Chauvet cave : available ${ }^{14} \mathrm{C}$ dates on cave bear skulls.

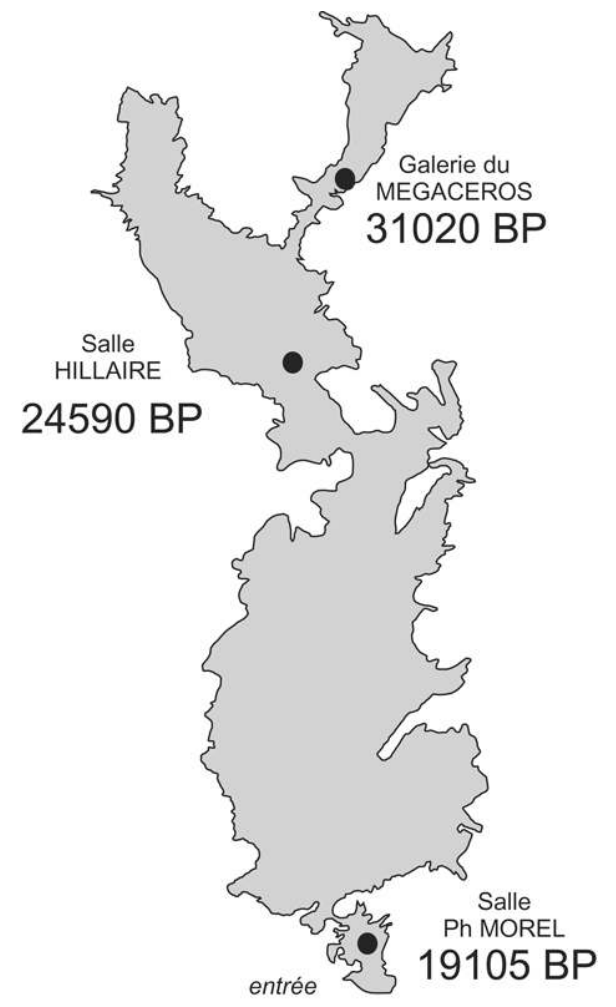

\section{2. Éléments de démographie}

Les crânes d'ours, abondants dans l'ensemble de la cavité, présentent toutes les phases de conservation (ou de destruction) que l'on peut rencontrer en contexte karstique. Dans certains secteurs (Salle des Bauges, Galerie des Mégacéros), les crânes qui reposent sur un sol argileux, sont parfois bien préservés (permettant l'identification de l'âge et $\mathrm{du}$ sexe) mais présentent parfois d'importantes destructions physicochimiques. Dans d'autres salles (Salle Hillaire, Salle du Crâne, Galerie des Croisillons), les crânes sont recouverts de calcite, d'épaisseur variable. A un encroûtement modéré des crânes succède un puissant enrobage faisant presque disparaître totalement le matériel paléontologique. C'est ce dernier stade de conservation qui prévaut pour la Salle Hillaire et la Salle du Crâne (69/88 échantillons). Seules les parties supérieures des crânes émergent des planchers stalagmitiques, les autres éléments squelettiques (éléments post-céphaliques), bien qu'éloignés des concentrations de crânes, sont enfouis dans la calcite. Très peu de crânes livrent par conséquent d'informations ostéométriques, permettant d'apprécier la variabilité intra populationnelle ${ }^{12}$, le sexratio ... Les crânes ne peuvent être mesurés en raison de leur emplacement 
(accessibilité, manipulation), position (beaucoup reposent sur leur face basale rendant impossibles les mesures importantes (longueur condylobasale, diamètre bizygomatique, diamètre transverse des canines)) ou encore de leur degré d'enfouissement (les trois quarts des crânes ne sont identifiés que par leur partie sommitale, ectoorbitaux ; encroûtement plus ou moins fort ...).

Les critères ostéomorphologiques (développement de la crête sagittale, bombement du frontal) suggèrent une nette sur-représentation des sujets femelles sur les mâles, dans toute la cavité ; le crâne sur le bloc rocheux de la salle du Crâne appartient à un individu subadulte femelle et présente des dimensions nettement inférieures aux crânes de mâles trouvés dans la Salle Brunel (ex. locus 3.2), le grand effondrement de la Salle Hillaire ou dans la partie Est de la Salle du Fond (locus 11.8). Pour les mêmes raisons, la structure en âges de ces taphodèmes ne peut être saisie; les individus très jeunes sont exceptionnels (un humérus dans l'amas 8.4 dans le secteur des Panneaux des Mains positives mesure moins de $4 \mathrm{~cm}$; une dizaine de dents lactéales isolées ont été repérées) et les juvéniles et sub-adultes (sutures crâniennes non fermées, denture définitive intacte, non usée) peu fréquents. Les adultes sensu lato sont les mieux représentés mais il est impossible de préciser la proportion de chaque tranche d'âge. La fonction de la grotte (station d'hivernation) est bien plus déterminée par la diversification des activités des ours que par leurs composantes démographiques (diachronisme partiel inter assemblages, cf. datations ${ }^{14} \mathrm{C}$ sur ossements).

La distribution des éléments squelettiques fait, elle aussi, apparaître toute la difficulté d'interprétation globale (par assemblage, intra et inter salles): aucune connexion anatomique importante n'est apparente ${ }^{13}$ et ce fort taux de désarticulation dans l'espace suppose une longue durée de fréquentation de la cavité par les ours, bouleversant les carcasses de leurs congénères, ainsi que des remaniements des dépôts existants par les agents non ursins (eau, homme?). Sans constituer la règle, les ensembles paléontologiques numériquement importants sont présents dans toutes les salles, sous forme d'enchevêtrements (secteur Ouest du grand effondrement de la Salle Hillaire (locus 9.3) ou sous forme d'épandages (secteur Ouest ( = entrée pléistocène + la “plage ") et fond (= loci $8.4-8.11$, au pied du panneau des Mains Positives jusqu'à l'entrée de la Salle Rouzaud) de la Salle des Bauges, fond de la Galerie des Croisillons ...).

\section{3 - Bioglyphes ${ }^{14}$}

Les traces laissées par les différentes activités ursines sont relativement fréquentes dans les cavités (cf. Viehmann 1973 ; Clot 1986...) mais encore peu étudiées. Dans la grotte Chauvet, de nombreux couchages (près de 300 au total) ont été répertoriés (fig. 13). Ces bauges sont particulièrement bien conservées dans la zone centrale de la Salle des Bauges, la Galerie du Cierge et dans la partie droite de la Salle Hillaire, abritée par l'imposante banquette d'argile, et dans les parties terminales de la cavité car éloignées (surélevés) des zones de circulation des eaux et des ours. Ces zones forment de véritables " dortoirs collectifs", les bauges se jouxtant les unes aux autres. Ces dépressions, bien que pouvant atteindre $1 \mathrm{~m}$ de diamètre et plusieurs dizaines de centimètres de profondeur, offrent en moyenne des dimensions très modestes (moins de $50 \mathrm{~cm}$ de diamètre et moins de $15 \mathrm{~cm}$ de profondeur). Aucun vestige osseux n'a été rencontré dans ces nids. 
Figure 13 - Grotte Chauvet : dénombrement et répartition spatiale des bauges d'ours des cavernes. Figure 13 - Chauvet cave: counting and spatial distribution of cave bear beds.

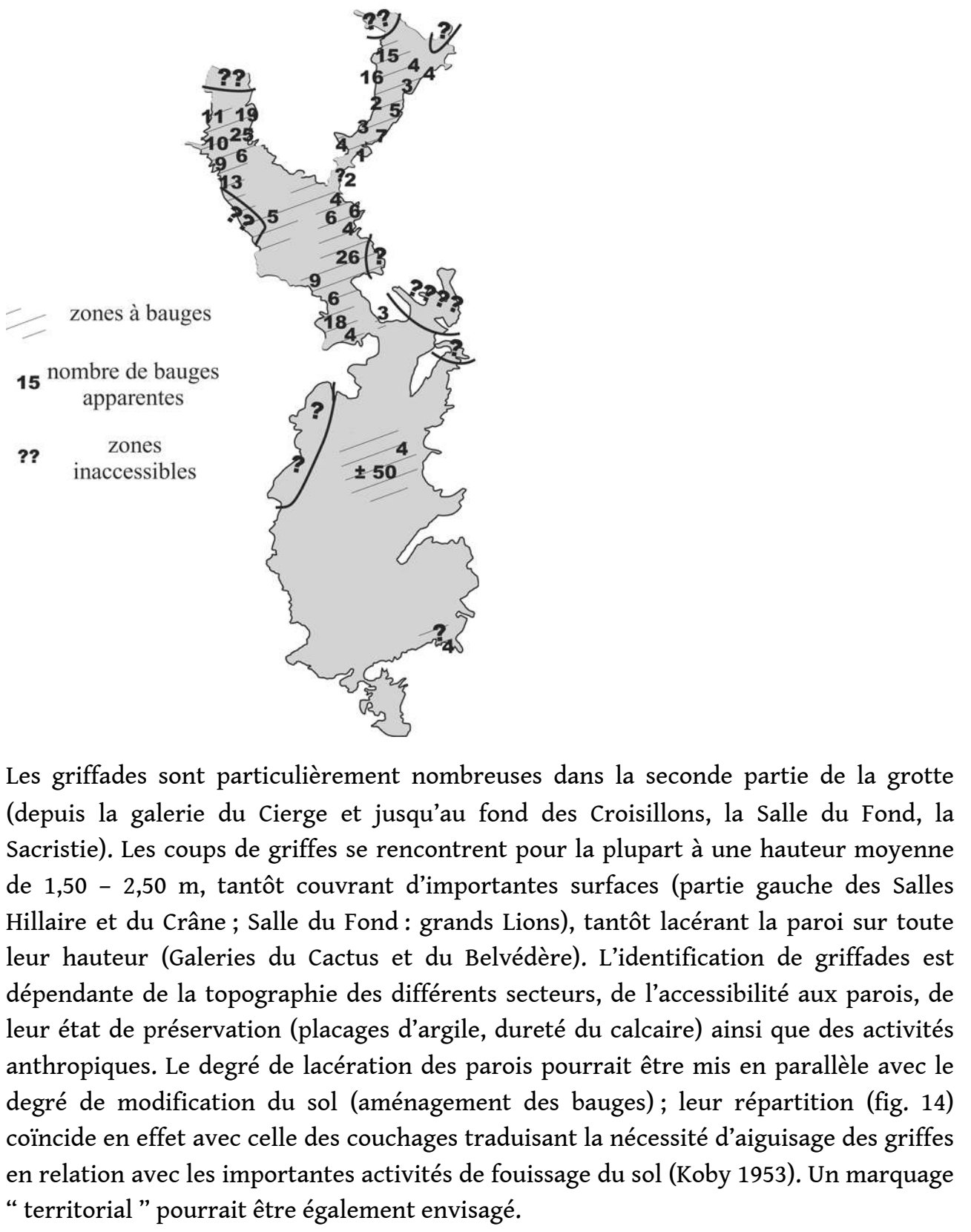


Figure 14 - Grotte Chauvet : répartition spatiale des griffades d'ours des cavernes. Figure 14 - Chauvet cave: spatial distribution of cave bear scratches.

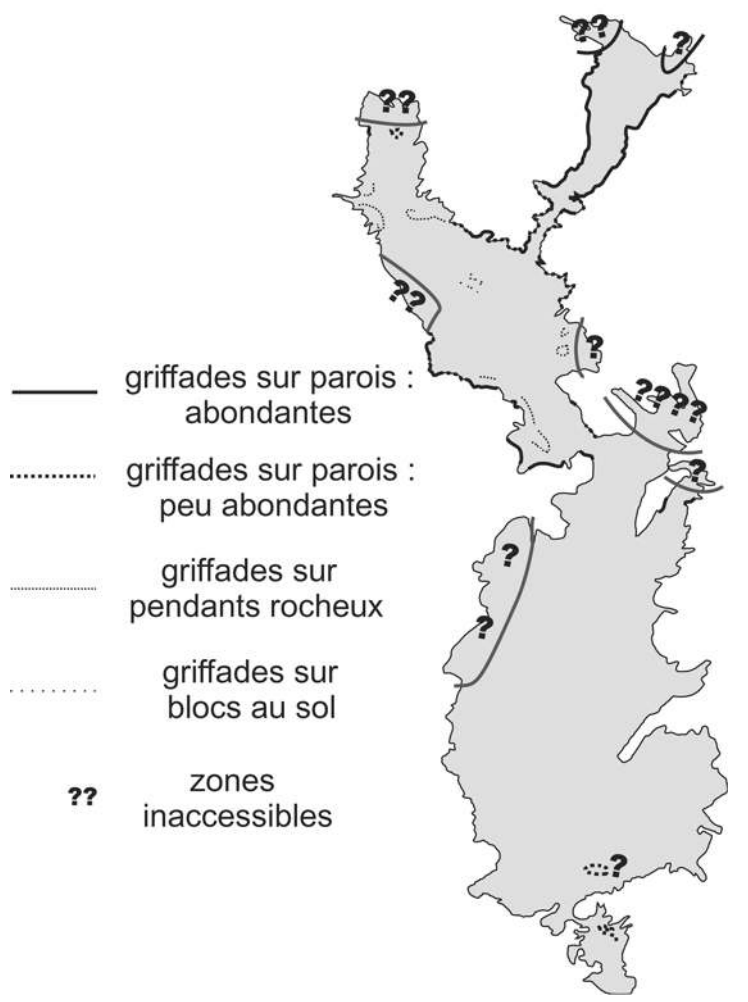

37 Les pieds de parois et les rebords des anfractuosités sont véritablement usés par les passages répétés des animaux, qui se déplacent dans l'obscurité principalement au toucher (" mémorisation topographique " des salles) et à l'odorat (marquage d'un territoire ?, carcasses de congénères en décomposition ?). Les polis ("Bärenschliffen " des auteurs germaniques), parfois difficiles à identifier (desquamation de la paroi) sont concentrés sous les panneaux de griffades et/ou les zones de passages (empreintes). Leur répartition est identique à celle des griffades (fig. 15). 
Figure 15 - Grotte Chauvet : répartition spatiale des polis d'ours des cavernes. Figure 15 - Chauvet cave: spatial distribution of polished walls made by cave bear.

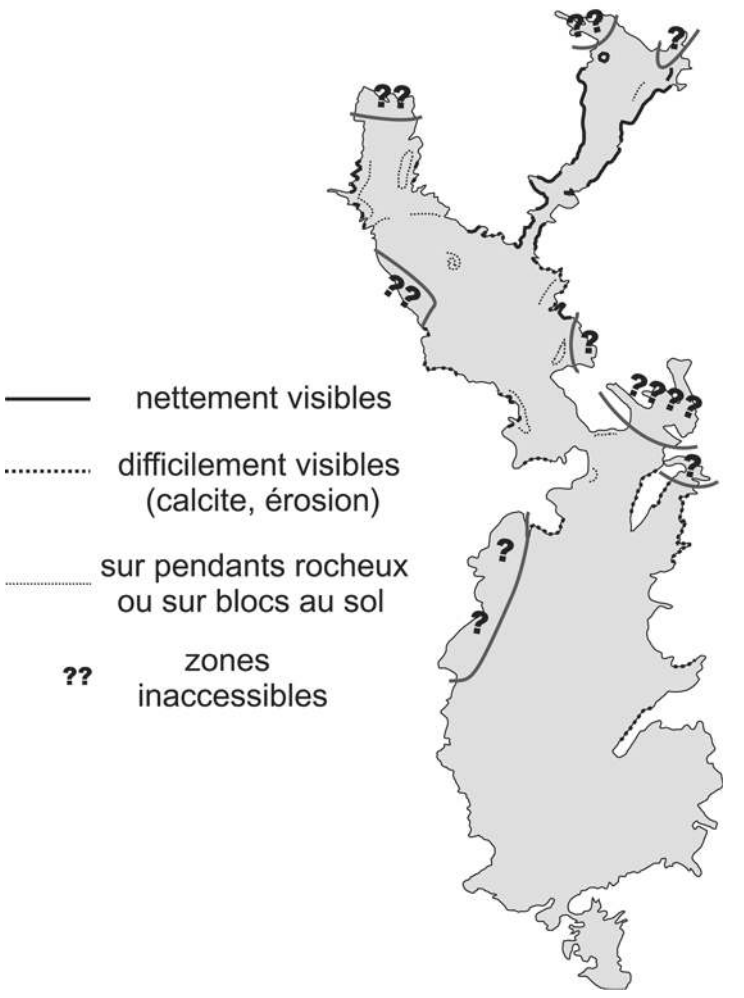

\section{3- Éléments d'anthropozoologie}

La disposition actuelle des ensembles paléontologiques est le résultat des activités combinées des ours, de l'homme et des agents naturels (eau, soutirages, enfouissements partiels...). La chronologie de chaque intervenant n'est, à ce jour, pas établie clairement pour l'ensemble des secteurs de la cavité mais il est certain que les Paléolithiques ont utilisé l'ours : sans reparler d'anthropozoologie au sens " alpin " du terme, force est de reconnaître que les interactions entre occupations ursines et humaines d'une part et surtout l'utilisation des premiers, au propre (ossements) comme au figuré (représentations ; cf. Baffier et Ferruglio 2001b) par les seconds est évidente, sans qu'il soit toujours possible d'étayer rationnellement ces observations.

Les actions humaines reconnues sur le stock osseux peuvent être organisées en différents gradients; le crâne d'ours sur le bloc rocheux de la Salle du Crâne constitue le principal critère d'une intervention anthropique, d'autres observations (" négatifs" d'au moins quatre os déplacés dans la Salle du Crâne, fémur dans la Salle Brunel, côte sur un surplomb rocheux près des Points rouges, vertèbre à la sortie de la Bretelle, côte au sommet de la paroi Ouest de la Galerie du Cactus) ne constituant que des indices d'une manipulation d'ossements. La Salle du Crâne présente l'étonnante singularité de contenir plus d'une cinquantaine de crânes d'ours (cinquante trois repérés à ce jour), dans un périmètre d'une dizaine de mètres au pied du gros bloc rocheux (sur lequel repose également un crâne d'ours). Il n'y a pas de positionnement préférentiel des crânes (certains reposent sur leur face basale, d'autres sont retournés ou de profil). Un épais lit de calcite ennoie souvent ces crânes, ne laissant émerger que les parties 
sommitales (zones frontales, crêtes sagittales, arrière-crânes). La prédominance des crânes sur tout autre ossement, dans ce secteur au moins, paraît évidemment intrigante. Même si une intervention de l'homme pouvait être envisagée, il convient d'insister sur l'action combinée de phénomènes non anthropiques (eau, charriage, ours) qui a modelé cette configuration particulière des vestiges osseux; le niveau d'ennoiement est visible sur plusieurs pièces (crânes au pied du gros bloc rocheux, crânes près de la ceinture argileuse Nord et Sud) et à la périphérie de cette salle, des zones non ou faiblement calcitées, contiennent d'importantes quantités d'éléments postcrâniens (os longs, côtes...). L'absence de ces vestiges " plats" dans la zone des crânes peut s'expliquer, à ce jour, tout aussi bien par l'épaisse calcitation recouvrant le sol que par une intervention anthropique. Préciser la chronologie, les circonstances et surtout les motivations qui ont régi cet assemblage serait prématuré, tant les conséquences, dans les différents champs de recherche en archéologie préhistorique sont importantes.

Deux autres catégories de témoins anthropiques avaient été identifiés lors des premières observations : os longs plantés dans le sol et os calcinés. Un examen plus attentif de ce matériel permet de privilégier une action non anthropique dans la mise en place de ces pièces.

41 Dans les premières salles (Salle des Bauges, Salle Brunel, Salle des Panneaux Rouges), d'importants amas d'ossements d'ours, résultant d'un charriage par les eaux, sont visibles. La " Plage " comprend ainsi plusieurs centaines d'ossements couvrant une longue bande de la partie gauche de la Salle des Bauges. Sur ses marges, en bordure de parois, deux humérus apparaissent plantés verticalement dans le sol argileux, à proximité de crânes d'ours. L'absence de présence humaine dans ce secteur (empreintes, activités domestiques) favorise l'hypothèse d'une action naturelle, d'autant que d'autres os longs, également enfoncés verticalement dans l'argile, apparaissent dans des anfractuosités toutes proches, à la base des battements d'eau (dépôt d'argile fine sub-horizontal).

Dans les foyers de la galerie des Mégacéros, des os d'ours calcinés avaient été repérés. Ces pièces (corps vertébraux principalement) sont enrobés de charbon de bois par imprégnation mais leur surface corticale demeure uniformément brune et la spongiosa est non affectée (aucune craquelure). L'utilisation de l'os d'ours comme combustible reste à démontrer.

\section{Conclusions}

La grotte Chauvet apparaît comme un remarquable exemple de grotte où l'Ours des cavernes a hiverné. Indépendamment de tous ses autres intérêts (spéléologiques, karstologiques, préhistoriques), avec les 190 crânes d'ours actuellement recensés et avec tous les autres éléments squelettiques et les autres espèces, cette cavité constitue un remarquable gisement paléontologique. L'inventaire des vestiges osseux (notamment les crânes) dans chacun des secteurs accessibles de la grotte et les différentes traces d'activités animales permettent d'établir les premiers jalons chronologiques des fréquentations ursines et anthropiques de la cavité : ours / homme / ours.

44 L'action combinée des hommes, des animaux cavernicoles et des agents naturels (écoulements d'eau, soutirages, calcitation) explique la disposition actuelle des 
ossements dans la grotte. De ce premier travail de relevés in situ, surgit toute la difficulté d'interpréter, dans l'espace et dans le temps, les intrications visibles en apparence entre l'homme paléolithique et l'Ours des cavernes. Les études futures auront pour but de déterminer ce qui revient aux uns et aux autres dans la formation et la modification des assemblages osseux, en relation avec les activités spécifiques pratiquées dans la cavité. Ces recherches auront pour objectif de caractériser les activités ursines et d'intégrer les données paléobiologiques (animales) à la géochronologie de la cavité.

Par son exceptionnelle richesse en témoins archéologiques et paléontologiques, la grotte Chauvet apparaît être un lieu de recherches scientifiques privilégiées (car novatrices) sur les comportements des groupes humains paléolithiques en relation avec leur environnement naturel.

\section{Remerciements}

Les auteurs adressent leurs plus vifs remerciements au Ministère de la Culture qui permet à l'ensemble de "l'équipe Chauvet" d'effectuer ces recherches dans les meilleures conditions. Les remarques des rapporteurs (Jean-Philip Brugal et Jean-Luc Guadelli) ont été constructives. Qu'ils soient ici remerciés.

\section{BIBLIOGRAPHIE}

ALTUNA J. 1973a - Distinción craneal entre la Marta (Martes martes) y la Foina (M. foina). Munibe, 25(1) : 33-38.

ALTUNA J. 1973b - Hallazgos de Oso Pardo (Ursus arctos, Mammalia) en cuevas del País Vasco. Munibe, 25/2-4 :121-170.

AUJOULAT N. et GUÉRIN C. 2001 - Les rhinocéros. In : Clottes J. (dir). : La Grotte Chauvet : L'art des origines. Paris, Seuil éditions : 174-176.

BAFFIER D. et FERUGLIO V. 2001a - Les cervidés. In : Clottes J. (dir). : La Grotte Chauvet : L'art des origines. Paris, Seuil éditions : 189-192.

BAFFIER D. et FERUGLIO V. 2001b - Les ours. In : Clottes J. (dir). : La Grotte Chauvet : L'art des origines. Paris, Seuil éditions : 192-193.

BALLESIO R. 1979 - Le gisement pléistocène supérieur de la grotte de Jaurens à Nespouls, Corrèze, France : les carnivores (Mammalia, Carnivora). 1. Canidae et Hyaenidae. Nouvelles Archives du Muséum d'Histoire Naturelle de Lyon, 17 : 25-55.

BOURDELLE E. et DEZILIERE M. 1949 - Notes ostéologiques et ostéométriques sur la tête de l'ours des Pyrénées dans le cadre de l'Ours brun en général (Ursus arctos L.). Mammalia, 12 : 125-128.

CAMARRA J.- J. 1989 - L'Ours brun. Paris, éds. Hatier, 325p.

CLOT A. 1980 - La grotte de la Carrière (Gerde, (Hautes-Pyrénées). Stratigraphie et Paléontologie des Carnivores. Thèse 3ème cycle, Université Paul Sabatier Toulouse, Laboratoire de Géologie, 237p. 
CLOT A. 1986 - Sur les traces des ours anciens. Les ours quaternaires pyrénéens, présentation générale. In : Dendaletche C. (Ed), L'Ours brun, Pyrénées, Abruzzes, Monts cantabriques, Alpes du Trentin (Biologie, Ecologie, Ethnographie, Protection), Acta Biologica Montana, 6 : 31-56.

CLOTTES J. 2001 - La Grotte Chauvet : L'art des origines. Paris, Seuil éditions.

CLOTTES J. et PACKER G. 2001 - Les félins. In : Clottes J. (dir). : La Grotte Chauvet : L'art des origines. Paris, Seuil éditions : 177-180.

CORDY J.M. 1972 - Etude de la variabilité des crânes d'Ours des cavernes de la collection Schmerling. Annales de Paléontologie, Vertébrés, LVIII : 151-207.

DESSE J., CHAIX L. et DESSE-BERSET N. 1986 - « Ostéo » ; base-réseau de données ostéométriques pour l'archéozoologie. Procédures, codages, exploitation des données, organisation du réseau. CRA, Notes et Monographies techniques $20: 161 \mathrm{p}$.

DONAT-AYACHE B. 1997 - Etude crânienne et dentaire de l'ours brun des sites karstiques du Mont Ventoux (Vaucluse). DEA Quaternaire : Géologie, Paléontologie Humaine, Préhistoire. Paris, Museum National d'Histoire Naturelle : 29p.

FOSSE Ph., JAUZION G., MAKSUD F., QUETTIER D., QUETTIER R., ROUCH Ph., et BESSON J.P. 2001 Ursidés pléistocènes des Pyrénées : éléments de paléontologie et de paléobiologie. Bull. Soc. Prehist. Ariège-Pyrénées, LVI : 103-138.

FOSSE Ph, BRUGAL J.P. et MOREL Ph. 2002 - Taphonomie et éthologie des ursidés pléistocènes. In : Tillet, T., Binford, L.R. (Eds), L'Homme et l'Ours, ERAUL, 100 : 79-101.

GARCIA M.A. 2001 - Les empreintes et les traces humaines et animales. In : CLOTTES J. (dir). : La Grotte Chauvet : L'art des origines. Paris, Seuil éditions : 34-43.

GELY B. 2001 - Les mammouths. In : CLOTTES J. (dir). : La Grotte Chauvet : L'art des origines. Paris, Seuil éditions : 180-182.

KOBY F. Ed. 1951 - L'omoplate d'Ursus spelaeus. Verhandlungen der naturforschendenGesellschaft in Basel, LXII : 1-23.

KOBY F. Ed. 1953 - Modifications que les Ours des cavernes ont fait subir à leur habitat. Premier Congrès International de Spéléologie, vol. 4, section $4:$ 15-27.

LAVILLE H., PIERRE-MARIE R.P. et PRAT F. 1972 - La caverne à ours du Plo del May (commune de Verdalle, Tarn). Sédimentologie et paléontologie ; étude préliminaire. Travaux et Recherches de la Fédération Tarnaise de Spéléo-Archéologie, 9 : 37-53.

LE GUILLOU Y. 2001 - Les boeufs musqués. In : Clottes J. (dir). : La Grotte Chauvet : L'art des origines. Paris, Seuil éditions : 194.

LE GUILLOU Y. et MAKSUD F. 2001 - Topographie. In : Clottes J. (dir). : La Grotte Chauvet : L'art des origines. Paris, Seuil éditions : XX

PHILIPPE M. 1993 - L'ours des cavernes de La Balme à Collomb ; l'un des plus fabuleux animaux contemporains de l'homme préhistorique. Mémoires et documents de la Société Savoyarde d'Histoire et d'Archéologie ; La Savoie avant l'histoire, 95 : 85-94.

PHILIPPE M. et FOSSE Ph. 2001 - Les ossements des animaux sur le sol de la grotte. In : CLOTTES J. (dir). : La Grotte Chauvet : L'art des origines. Paris, Seuil éditions : 51-56.

PRAT F. et THIBAULT C. 1976 - Le gisement de Nauterie à La Romieu (Gers). Fouilles de 1967 à 1973. Nauterie I. Museum National d'Histoire naturelle (F), n.s., série C, Sciences de la Terre, Mémoires, $35: 43-82$. 
RODE P. et DIDIER R. 1944 - Différence entre la tête osseuse de la martre et celle de la fouine. Mammalia, 8(3-4) : 127-132.

SUIRE C. 1969 - Contribution à l'étude du genre Canis d'après les vestiges recueillis dans quelques gisements pléistocènes du Sud-Ouest de la France, Université de Bordeaux, thèse. 2 vols.

TORRES PEREZ-HIDALGO (de) T. 1988 - Osos (Mammalia, Ursidae) del Pleistoceno de laPenínsula Ibérica. Publicaciones especiales del boletín geológico y minero, 316p.

TSOUKALA E. et GRANDAL D'ANGLADE A. 2002 - Système de mesures sur le squelette d'Ursidae. In : Tillet, T., Binford, L.R. (Eds), L'Homme et l'Ours, ERAUL, 100 : 265-287.

VIEHMANN I. 1973 - Les traces de vie de l'ours des cavernes (Ursus spelaeus) dans les grottes de Roumanie. Institut de Spéléologie « Emile Racovitza » Livre du Cinquantenaire : 451-461.

\section{NOTES}

1. Travail effectué par l'équipe de G. Perazzio.

2. Déterminations P. Mein.

3. D'après les “Tableaux récapitulatifs des espèces animales et végétales inventoriées" de la Réserve Naturelle des Gorges de l'Ardèche ; décembre 1998, document non publié.

4. Déterminations P. Mein.

5. Déterminations C. Mourer.

6. Identification signalée dans les notes de Ph. Morel.

7. Information inédite de M. Philippe.

8. Données manuscrites de Philippe Morel

9. Aucun élément osseux n'a été sorti pour détermination mais, d'après les photos prises sur place et malgré la pellicule calcitique qui recouvre les ossements, C. Mourer a identifié l'Aigle royal.

10. Déterminations de C. Mourer.

11. Galerie des Mégacéros : $31020 \pm 350$ BP-Gifa 99773) ; Salle Hillaire : $24590 \pm 190$ BP (Ly 235) ; Salle Philippe Morel :19105 \pm 150 BP (Ly 1656 oxa).

12. Le second secteur de la cavité (depuis la galerie du Cierge jusqu'aux Croisillons et la Sacristie), a-t-il été occupé antérieurement à la première moitié de la cavité (salle Ph. Morel - salle des Bauges/Mains positives, galerie F. Rouzaud)?

13. Sensu paléontologique, c'est-à-dire taphodème (Cordy 1972).

14. Seules deux colonnes vertébrales isolées dans la Salle Hillaire, une extrémité de patte (métapodes + phalanges) sous un bourrelet argileux dans la Salle du Crâne, près du panneau des Chevaux et quelques éléments du squelette appendiculaire dans la Galerie des Croisillons ont été retrouvés en connexion anatomique stricto sensu.

\section{RÉSUMÉS}

Au-delà des quelque 450 peintures et gravures datées du Paléolithique supérieur ancien, la grotte Chauvet (Vallon-Pont-d'Arc, Ardèche) a livré d'abondants vestiges paléontologiques (près de 
4000 ossements jonchent le sol des différents secteurs de la cavité) ainsi qu'une riche palette de bioglyphes, d'origine animale (bauges, pistes, griffades et polis en pied de paroi) ou humaine (tracés digités, empreintes). Un premier inventaire de la faune identifiée (ossements sur les sols) souligne l'extrême prédominance de l'Ours des cavernes (99,9\% de la faune). Les interactions entre l'Homme et l'Ours sont présentées et discutées brièvement.

In addition to the 450 paintings and engravings dated to the early Upper Paleolithic, Chauvet cave (Vallon Pont d'Arc, Ardèche) yields numerous paleontological remains (almost 4000 bones have been found on the floors in all parts of the cavity) and a high diversity of bioglyphs, either of an animal origin (beds, tracks, scratches, polished walls) or of a human one (finger traces, footprints). A first survey allows to establish the predominance of the cave bear (99,9\%) of the faunal remains. Interactions between Man and Bear are also presented and discussed briefly.

\section{INDEX}

Keywords : Chauvet cave, fauna, cave bear, taphonomy

Mots-clés : grotte Chauvet, faune, Ours des cavernes, taphonomie

\section{AUTEURS}

\section{MICHEL PHILIPPE}

Pôle Sciences et Sociétés (Muséum d'Histoire Naturelle), Centre de Conservation et d'Etude des

Collections, 13A rue Bancel, 69007 Lyon. mail : michel.philippe@rhone.fr

\section{PHILIPPE FOSSE}

CNRS, Université de Toulouse-Le Mirail, UMR 5608 (UTAH), 5 allées Antonio-Machado, 31058

Toulouse cedex 1. mail : fosse@univ-tlse2.fr 